\title{
Femtocells Underlaid Macro-cellular Networks
}

\author{
Joydev Ghosh*, Debasis Das ${ }^{\dagger}$ \\ *School of Computer Science and Robotics, National Research Tomsk Polytechnic \\ University, Russia, Email: joydev.ghosh.ece@gmail.com \\ ${ }^{\dagger}$ Indian Institute of Technology(IIT) Jodhpur, India, Email: debasis@iitj.ac.in
}

\begin{abstract}
This work presents the evaluation of the downlink (DL) performance of a dual-layer cellular networks by using energy efficiency (EE) metric, where femto base stations (FBSs), macro base stations (MBSs) and users (FUs) form independent spatial Poisson point processes (PPPs). The proposed network model is developed by considering number of antennas at each BS alongside a single antenna at each user with the use of the conventional spectrum re-utilization approach. Then, Coverage probability and EE expressions for the duallayer cellular networks are exclusively derived analytically. It is also demonstrated that simulation results are almost in-line with the analytical one in the PPP-based model. While coverage probability deteriorates with less margin in the lower FBS density region compared to the scheme presented in [10] signalled not much turnaround of the network performance, $\mathrm{EE}$ in the lower and the upper FBS density regions are likely to remain between $6 \times 10^{-3}$ to $9.2 \times 10^{-3} \mathrm{Bits} / \mathrm{Joule}$ and $4.6 \times 10^{-3}$ to $7.1 \times 10^{-3}$ Bits/Joule, respectively. Proposed scheme tells us that it is firmly on course to match up with Vehicular Ad-hoc NETworks (VANET) applications without incurring high cost as EE, low latency, coverage probability and low power adaptability are back on good growth path.
\end{abstract}

Index Terms-Spatial Poisson Point Process; Femtocell; Energy Efficiency, Coverage probability

\section{INTRODUCTION}

Rapidly increasing energy cost and high demand of data traffic have motivated to articulate more on energy efficiency (EE) aspect of wireless networks [1]. Integrating cognitive radio (CR) with femtocell can further optimize network performance [2], [3]. Nowadays in telecommunications, a femtocell is a small base station with small network coverage and have developed to apply usually at a home or small business. The small-cell, with femto Access Point (FAP) as a subset, is more comprehensively used term in the industry. This links to the service provider's network via broadband (such as digital subscriber line (DSL) or cable). A femto base station (FBS) permits network providers to extend service coverage at indoors or at the cell edge, particularly where quality of service (QoS) would otherwise be limited or unavailable. [4], [5]. Femtocell decreases energy consumption which in turn prolong battery life of handsets [6], [7].

Here the system model has been showing in contrast to the reference [8] that the networks where the arrangements of the distribution has a high level of interrelationship with the random spatial model produced as a spatial Poisson point processes (PPPs), even for wide-reaching macrocells. The coverage probability $(\mathrm{CP})$ and $\mathrm{EE}$ of a cellular network consisting of FBSs only presented in [8] has been extended by analytically demonstrating further the networks with the co-existence scenario of macro base station (MBS) and FBSs. With this major extention of the network model and establishment of $\mathrm{EE}$ and $\mathrm{CP}$ expressions therein, we validate the proposed networks on its performance and it can be a good replacement of Road Side Units (RSUs) in a vehicular network with cellular infrastructure as a backbone [9].

The different models for the EE analysis of heterogeneous $\mathrm{CR}$ networks have been introduced in past, for example, the well-known Wyner and grid schemes [10]. The Wyner scheme is generally utilized because of its tractability but it may fail to keep sight of the important features of next generation wireless networks. The grid scheme is not much tractable particularly in the network where femtocell distribution is random in nature.

The twofold contributions of the work are as follows: we propose a novel set of analytical results by applying the spatial PPP model to compute EE of a dual-layer HetNets in association with multi-antenna base stations. Here, a novel set of analytical results have been presented by applying the spatial PPP model to compute EE of femto- 


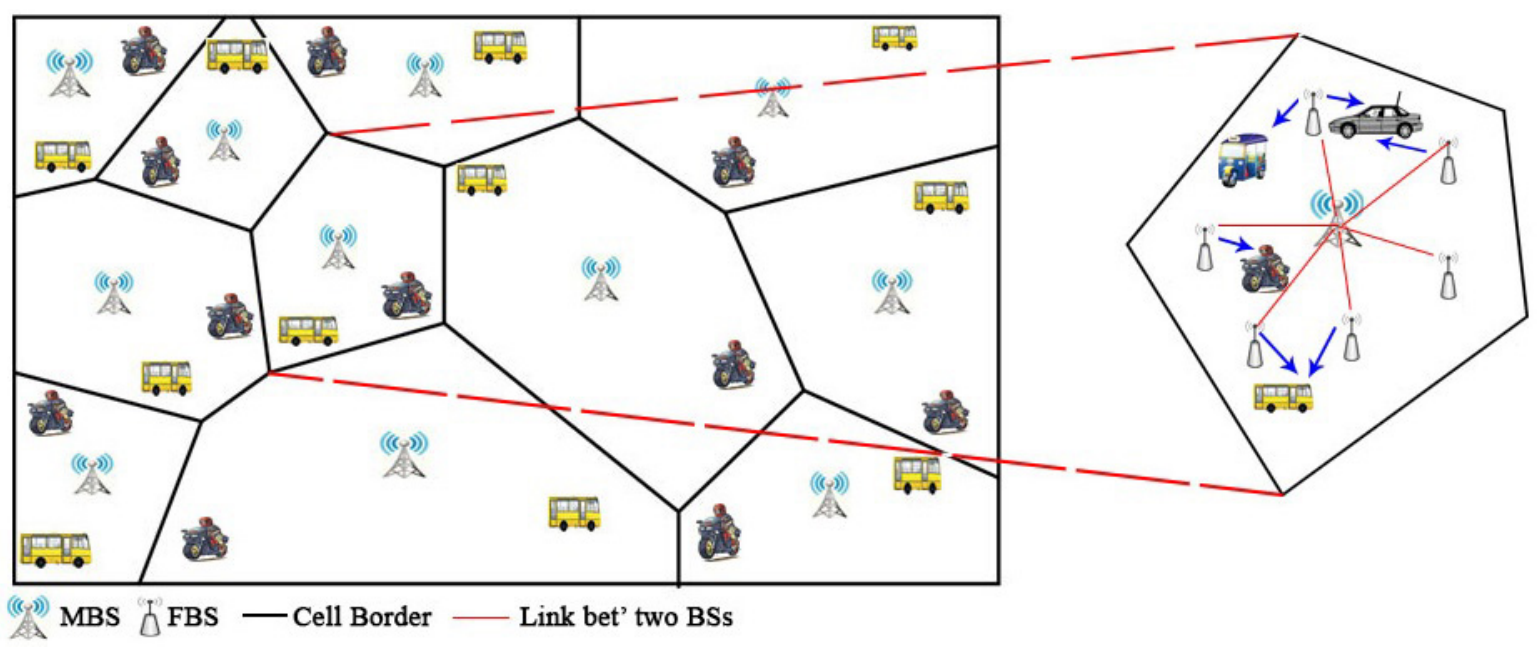

Fig.1: A two-tier heterogeneous cellular network model, where MBSs, FBSs and users are modeled as independent spatial PPPs.

cellular networks in association with multi-antenna base stations. We then demonstrate that the $\mathrm{EE}$ level of single-antenna type BSs is higher than the multiple-antenna type BSs only for the case whilst the power consumes by the analog circuit is higher as compared to the threshold (i.e., $P_{c p} \geq \bar{\gamma} P_{n t p}$ ), whereas BSs of multiple-antenna types are superior for the case whilst the power consumes by the analog circuit is less as compared to the threshold (i.e., $\left.P_{c p}<\bar{\gamma} P_{n t p}\right)$. Here, $P_{c p}$ indicates circuit power consumption, $P_{n t p}$ accounts for the non-transmit power and $\bar{\gamma}$ stands for the predefined threshold.

The organization of this paper is structured as follows. Section II describes the analytical framework of energy efficiency in femto-cellular networks. The numerical results have been presented in Section III where the impact on the EE has explained and achieved the useful insights for exploiting a single antenna or multiple antennas at the FBS. Finally, this work is ended up in Section IV with the conclusion. The key notations and their descriptions used in the paper are listed in Table I.

\section{System Model}

Fig. 1 presents a dual-layer network, where MBSs, FBSs, and Users are coexisting and modeled as independent spatial PPPs in twodimensional Euclidean plane denoted by $|\mathbf{R}|^{2}$, indicated as $\phi_{M B S}, \phi_{F B S}$ and $\phi_{U s e r}$, respectively. This network can be considered as a condensed deployment of MBSs and FBSs, where MBS, FBS and User densities are denoted by $\lambda_{M B S}$,
Table I: The notations of main network parameters

\begin{tabular}{|l|l|}
\hline Symbol & Description \\
\hline$\lambda_{M B S}$ & MBS density \\
\hline$\lambda_{F B S}$ & FBS density \\
\hline$\lambda_{U s e r}$ & User density \\
\hline$\epsilon$ & FBS to the user density ratio \\
\hline$p$ & Probability of the active FBSs \\
\hline $\bar{p}$ & Probability of the inactive FBSs \\
\hline $\mathbf{I}$ & Identity matrix \\
\hline$M$ & Number of antennas elements \\
\hline$P_{n t p}$ & Non-transmited power \\
\hline$T_{N}$ & Network throughput \\
\hline$A_{M}$ & Lower triangular Toeplitz matrix \\
\hline $\bar{\gamma}$ & SINR threshold \\
\hline$h_{j j}^{H}$ & Conjugate transpose of the matrix $h_{j j}$ \\
\hline$\left\|A_{M}\right\|_{1}$ & $\begin{array}{l}\max _{j=1: n} \sum_{i=1}^{M}\left|A_{i j}\right| \text { for maximal column } \\
\text { sum }\end{array}$ \\
\hline
\end{tabular}

$\lambda_{F B S}$ and $\lambda_{U s e r}$, respectively. Here, the downlink (DL) transmission is exclusively focused on, where network service is facilitated to the User either from the nearest FBSs or MBSs. This type of system model is absolutely perfect for the dual-layer cellular networks, where both shape of any types of cells and deployment of BSs are non-uniform. There can be two types of FBSs based on whether they can transmit signals or not as follows: inactive and active FBSs. Quite a few FBSs would not have the scope to provide service to Users due to the independent locations of users are known as inactive FBSs as opposite to active one. The probability of the active and the inactive FBSs can be denoted by $p$ and $\bar{p}$, respectively. Also, it is 
shown in [8], $p$ as a function of FBS to the user density ratio denoted by $\epsilon$, where $\epsilon \triangleq \frac{\lambda_{F B S}}{\lambda_{u s e r}}$. Now, $\bar{p}$ can further be re-expressed using [8], [12] as:

$$
\begin{aligned}
\bar{p}=\left(1+\frac{1}{\beta \epsilon}\right)^{-\beta} & \approx\left(1-\frac{\beta}{\beta \epsilon}\right) \\
& \approx\left(1-\frac{1}{\epsilon}\right), \frac{\lambda_{F B S}}{\lambda_{\text {user }}} \rightarrow \infty
\end{aligned}
$$

where, $\beta$ is a constant in regard to the femtocell size distribution achieved through data fitting [12].

Therefore, $p=1-\bar{p}=\frac{\lambda_{\text {user }}}{\lambda_{F B S}}$.

The signal-to-interference plus noise ratio (SINR) measured at a particular instant for a femtocell user can be expressed by:

$$
\begin{aligned}
& \gamma=\frac{P_{t p 1} g_{j j} d_{j j}^{-\alpha}}{\sum_{k \in \bar{\phi}_{F B S}} P_{t p 1} g_{k j} d_{k j}^{-\alpha}} \\
& +\sum_{l \in \bar{\phi}_{M B S}} P_{t p 2} g_{l j} d_{l j}^{-\alpha}+N_{0}
\end{aligned}
$$

where $P_{t p 1}$ and $P_{t p 2}$ indicate the transmit power of femtocell and macrocell at the transmitter and $N_{0}$ stands for the additive white gaussian noise (AWGN) power; $d_{j j}, d_{k j}$ and $d_{l j}$ represent separation between $j^{\text {th }}$ active FBS to $j^{\text {th }}$ FU, $k^{t h}$ active FBS to $j^{\text {th }} \mathrm{FU}$ and $l^{\text {th }} \mathrm{MBS}$ to $j^{\text {th }} \mathrm{FU}$, respectively; $\alpha$ stands for the pathloss exponent; $\bar{\phi}_{M B S} \bar{\phi}_{F B S}$ denote the set of MBSs and active FBSs; $g_{k j}$ and $g_{l j}$ represent the channel gain between $k^{\text {th }}$ active FBS to $j^{\text {th }} \mathrm{FU}$ and between $l^{\text {th }}$ MBS to $j^{\text {th }} \mathrm{FU}$, i.e., $g_{j j}=\left\|h_{j j}\right\|^{2}$, $g_{k j}=\left|\frac{h_{j j}^{H}}{\left\|h_{j j}\right\|} h_{k j}\right|^{2}$ for $k \neq j$, and $g_{l j}=\left|\frac{h_{j j}^{H}}{\left\|h_{j j}\right\|} h_{l j}\right|^{2}$ for $l \neq j$ where $h_{k j} \sim \mathcal{C N}(0, \mathbf{I})$ and $h_{l j} \sim \mathcal{C N}(0, \mathbf{I})$ are the complex-valued Gaussian random variable which represents the channel fading between $k^{\text {th }}$ active FBS to $j^{\text {th }} \mathrm{FU}$, and I stands for the identity matrix [1]. Here, we mainly focus on the most important performance metric, EE which can be determined by means of the outage probability. We can also not underestimate the role of the network throughput and power consumed by the BSs to compute the EE. An under-privileged user are treated as the outage and it can be determined by $p_{\text {outage }}=P_{r}(\gamma \leq \bar{\gamma})$. Thus, the network throughput can be expressed by applying (1):

$$
\begin{aligned}
T_{N} & =p \lambda_{F B S}\left(1-p_{\text {outage }}\right) \log _{2}(1+\bar{\gamma}) \\
& =\lambda_{\text {user }}\left(1-p_{\text {outage }}\right) \log _{2}(1+\bar{\gamma}),
\end{aligned}
$$

where $p \lambda_{F B S}$ denotes the density of active FBSs, $p \lambda_{F B S}\left(1-p_{\text {outage }}\right)$ represents the successful transmission of data to the FUs located in the networks and $\log _{2}(1+\bar{\gamma})$ stands for constant transmission rate. The power consumed by the BSs can be expressed by following the linear equations as in [10]:

$$
\begin{aligned}
P_{P C}=\frac{1}{\eta_{A E 1}} P_{t p 1}+\frac{1}{\eta_{A E 2}} P_{t p 2} & +M\left(P_{c p 1}+P_{c p 2}\right) \\
+ & P_{n t p},
\end{aligned}
$$

where $\eta_{A E 1}$ and $\eta_{A E 2}$ denote the power amplifier efficiency for FBS and MBS respectively; $P_{c p 1}$ and $P_{c p 2}$ indicate circuit power consumption for femtocell and macrocell respectively; $P_{n t p}$ accounts for the non-transmitted power and $M$ stands for the number of antenna elements. Now, by considering the FBS power model similar to [13] into account, (4) can further re-express the average power consumption per unit area as:

$$
\begin{aligned}
P_{A P C} & =\lambda_{u s e r}\left[\frac{1}{\eta_{A E 1}} P_{t p 1}+M P_{c p 1}\right] \\
& +\lambda_{M B S}\left[\frac{1}{\eta_{A E 2}} P_{t p 2}+M P_{c p 2}\right] \\
& +\left(\lambda_{F B S}+\lambda_{M B S}\right) P_{n t p},
\end{aligned}
$$

where 1st part and 2nd part of (5) signifies the power consumed by the active FBSs and MBS rest part of (5) accounts for the non-transmit power from both active and inactive BSs. The network EE can be expressed by [11]:

$$
\eta_{E E}=\frac{T_{N}}{P_{A P C}}
$$

Here, the maximal ratio transmission $(\mathrm{MRT})^{1}$ or space-time processing transmit diversity schemes are used at each multi-antenna FBS [15]. The cumulative distribution function (CDF) of $g_{j j}$ for

\footnotetext{
${ }^{1}$ Note that an extension of this paper is also applicable for different multi-antenna reception schemes, where interference distribution will be the major change that will amend the current analytical expressions.
} 
$g_{j j} \sim \Gamma(M, 1)$, we get [14] based on (2):

$$
\begin{aligned}
p_{\text {outage }} & =P_{r}\left(P_{t p 1} g_{j j} \leq d_{j j}^{\alpha} \bar{\gamma}\left(I_{f}+I_{m}\right)\right) \\
& =1-E_{d_{j j}}\left[E_{I}\left[\sum_{n=0}^{M-1} \frac{d_{j j}^{\alpha n}}{n !} I^{n} e^{-d_{j j}^{\alpha n}}\right]\right],
\end{aligned}
$$

where

$$
\begin{aligned}
I & \triangleq \bar{\gamma}\left(\sum_{k \in \phi_{F B S}^{-}} P_{t p 1} g_{k j} d_{k j}^{-\alpha}+\sum_{l \in \phi_{M B S}^{-}} P_{t p 2} g_{l j} d_{l j}^{-\alpha}\right) \\
& \triangleq \bar{\gamma}\left(I_{f}+I_{m}\right) .
\end{aligned}
$$

For $s_{n} \triangleq d_{j j}^{a n}, E_{I}\left[e^{-s_{n} I}\right]$ is the interpretation of Laplace Transformation (LT) of $I, \mathcal{L}(\cdot)$. Thus, $E_{I}\left[I^{n} e^{-s_{n} I}\right]=(-1)^{n} \frac{d_{n}}{d S_{n}} \mathcal{L}(s)$. Hence, (7) can be further written as:

$$
p_{\text {outage }}=1-E_{d_{j j}}\left[E_{I} \sum_{n=0}^{M-1} \frac{s_{n}}{n !}(-1)^{n} \frac{d_{n}}{d s^{n}} \mathcal{L}(s)\right] \text {. }
$$

The main difficulties with the derivation in (8) is to deal with the $n^{\text {th }}$ derivation of $\mathcal{L}(s)$. The closedform relation of $n$-th derivative can be converted into the form of a lower triangular Toeplitz matrix (LTTM) that will help to quantify the analytical properties to evaluate the network performance. Therefore, outage probability can be re-expressed in the given theorem.

Theorem $1: p_{\text {outage }}=1-\frac{1}{p}\left\|\left[\left(a_{0}+\frac{1}{p}\right) \mathbf{I}-A_{M}\right]^{-1}\right\|_{1}$ where $\mathbf{I}$ accounts for an $M \times M$ identity matrix. A LTTM, denoted by $A_{M}$, and its $L_{1}$ norm $\left\|A_{M}\right\|_{1}$ has the following properties:

i) $A_{M}$ with positive entries can be expressed as:

$$
A_{M}=\left[\begin{array}{ccccc}
a_{0} & & & & \\
a_{1} & a_{0} & & & \\
a_{2} & a_{1} & a_{0} & & \\
\vdots & & & \ddots & \\
a_{M-1} & a_{M-2} & \cdots & a_{1} & a_{0}
\end{array}\right]
$$

where, $a_{n}>0$ for $n \in[0, M-1]$ and the closedform relation is expressed in (10).

ii) $\left\|A_{M}\right\|_{1}$ is bounded as

$$
\frac{1}{1+p X_{l}} \leq\left\|A_{M}\right\|_{1} \leq \frac{1}{1+p X_{u}},
$$

where, $X_{l}=x_{j}-\sum_{k=1}^{M-1}\left(1-\frac{k}{M}\right) x_{k}, X_{u}=x_{j}$
$\sum_{k=1}^{M-1} x_{k}, X_{l}$ and $X_{u}$ are independent of both $\lambda_{F B S}$ and $\lambda_{\text {user }}, x_{j}=\frac{\frac{2}{\alpha} \bar{\gamma}}{1-\left(\frac{2}{\alpha}\right)}{ }_{2} F_{1}\left(1,1-\frac{2}{\alpha} ; 2-\frac{2}{\alpha} ;-\bar{\gamma}\right)$, and $x_{k}=\frac{\frac{2}{\alpha} \bar{\gamma}^{k}}{k-\frac{2}{\alpha}} 2 F_{1}\left(k+1, k-\frac{2}{\alpha} ; k+1-\frac{2}{\alpha} ;-\bar{\gamma}\right)$ for $k \geq 1$, where ${ }_{2} F_{1}($.$) accounts for Gauss hy-$ pergeometric function.

Proof : The proof of $p_{\text {outage }}$ in terms of $A_{M}$ is referred to Appendix A presented in [8].

The $L_{1}$ norm of a LTTM, $\left\|A_{M}\right\|_{1}$, can be expressed in relation to (8) as: $\left\|A_{M}\right\|_{1}=$ $\max _{1 \leq j \leq n} \sum_{i=1}^{M}\left|A_{i j}\right|$ for $A_{M} \in R^{M \times n}$.

The recurrence relation of $a_{n}$ is provided in [16] as $a_{n}=v \sum_{k=0}^{n-1} x_{n-k} a_{k}$, where $a_{0}=\frac{1}{1+x_{0} p}$ and $v=\frac{p}{1+x_{0} p}$. The closed-form relation of $a_{n}(n \geq 1)$ can be expressed as:

$$
\begin{array}{r}
a_{n}=\frac{1}{1+x_{0} p} \sum_{k=1}^{n} v^{k} \sum_{\begin{array}{c}
k \\
k_{1}+\cdots+k_{n}=k \\
k_{1}+\cdots+n k_{n}=n
\end{array}}\left(\begin{array}{ccc}
k \\
k_{1} & \cdots & k_{n}
\end{array}\right) \\
\left(\frac{x_{1}}{x_{0}}\right)_{1}^{k} \cdot\left(\frac{x_{n}}{x_{0}}\right)_{n}^{k}
\end{array}
$$

To find an upper limit of $\left\|A_{M}\right\|_{1}$, we re-express $\left\|A_{M}\right\|_{1}=v / p\left\|\left(\mathbf{I}-v B_{M}\right)^{-1}\right\|_{1}$, where $\mathbf{I}$ accounts for an $M \times M$ identity matrix and $B_{M}$ stands for an $M \times M$ Toeplitz matrix, i.e.,

$$
B_{M}=\left[\begin{array}{ccccc}
x_{0} & & & & \\
x_{1} & x_{0} & & & \\
x_{2} & x_{1} & x_{0} & & \\
\vdots & & & \ddots & \\
x_{M-1} & x_{M-2} & \cdots & x_{1} & x_{0}
\end{array}\right]
$$

Since $\left(\mathbf{I}-v B_{M}\right)\left(\mathbf{I}-v B_{M}\right)^{-1}=\mathbf{I}$, we get:

$$
\left(\mathbf{I}-v B_{M}\right)^{-1}=\mathbf{I}+v B_{M}\left(\mathbf{I}-v B_{M}\right)^{-1}
$$

Then, applying the triangle inequality, we get:

$$
\left\|\left(\mathbf{I}-v B_{M}\right)^{-1}\right\|_{1} \leq\|\mathbf{I}\|_{1}+v\left\|B_{M}\right\|_{1}\left\|\left(\mathbf{I}-v B_{M}\right)^{-1}\right\|_{1},
$$

and this is expressed by $\left\|\left(\mathbf{I}-v B_{M}\right)^{-1}\right\|_{1} \leq$ $\|I\|_{1} /\left(1-v B_{M}\right)_{1}$. As $\|\mathbf{I}\|_{1}=1$, and $\left\|B_{M}\right\|_{1}=\sum_{k=1}^{M-1} x_{k}$, hence we get an upper 
limit of $\left\|A_{M}\right\|_{1}$ as

$$
\begin{aligned}
\left\|A_{M}\right\|_{1} & \leq \frac{1}{\left(1+x_{0} p\right)\left(1-v \sum_{k=1}^{M-1} x_{k}\right)} \\
& =\frac{1}{1+p\left(x_{0}-\sum_{k=1}^{M-1} x_{k}\right)} .
\end{aligned}
$$

To find lower limit of $\left\|A_{M}\right\|_{1}$, we consider $a \triangleq$ $(1,1, \cdot \cdot 1)^{T}$ with $b \triangleq \mathbf{U} a$ where $\mathbf{U}$ is an invertible matrix, hence we can express $a=\mathbf{U}^{-1} b$. Applying the inequality $\|a\|_{1} \leq\|\mathbf{U}\|_{1}\|b\|_{1}$, we get $\|\mathbf{U}\|_{1} \geq$ $\frac{\|a\|_{1}}{\|b\|_{1}}$. Since $b=\mathbf{U} a$, we get $\|b\|_{1}=M\left(x_{0}+\frac{1}{p}\right)-$ $(M-1) x_{1}-\cdots-x_{M-1}$. Hence, we can get lower limit of $\left\|A_{M}\right\|_{1}$ as:

$$
\begin{array}{r}
\|\mathbf{U}\|_{1} \geq \frac{1}{p} \frac{\|a\|_{1}}{\|b\|_{1}}=\frac{1}{1+p\left(x_{0}-\sum_{k=1}^{M-1} x_{k}\right.} . \\
\left.+\sum_{k=1}^{M-1} \frac{k}{M} x_{k}\right)
\end{array}
$$

Point to be noted that $x_{k}>x_{k+1}$ for $k \in \mathcal{N}$, and $\sum_{k=1}^{\infty} x_{k}=x_{0}$. Therefore, both $X_{l}$ and $X_{u}$ are positive, $X_{l}-X_{u}=\sum_{k=1}^{M-1} \frac{k}{M} x_{k}$ is a function of $M$ and the difference value is a decreasing function while $M$ is higher than a specific preset value.

Lemma 1: The coverage probability is bounded as

$$
\frac{1}{1+p X_{l}} \leq p_{\text {cov }} \leq \frac{1}{1+p X_{u}}
$$

where $X_{l}$ and $X_{u}$ are defined in Theorem 1.

Proof : As $p_{\text {cov }}=\left\|A_{M}\right\|_{1}$, this property follows Theorem 1. Hence, $p_{c o v}$ can be approximated from (10) as:

$$
p_{\text {cov }}=\left\|A_{M}\right\|_{1} \approx \frac{1}{1+p X}
$$

where $X$ lies between $X_{l}$ and $X_{u}$. The above approximation differentiates the effect of $\lambda_{B S}$ and $M$, respectively, as $p$ depends on $v$, whereas $X_{l}$ and $X_{u}$ can be determined by $M, \alpha$, and $\bar{\gamma}$. The consequences of the BS density is mainly articulated in this property.

Lemma 2: With the increasing amount of transmit antennas from $M$ to $M+1$, the performance gain with regard to the coverage probability can be given by:

$$
p_{\text {cov }}(M+1)-p_{\text {cov }}(M)=a_{M},
$$

where, $p_{\text {cov }}(M)$ indicates the coverage probability with $M$ transmit antennas at individual BS.

Proof : The analytical result can be extracted by the following equality

$$
p_{\text {cov }}(M)=\left\|A_{M}\right\|_{1}=\sum_{n=0}^{M-1} a_{n} .
$$

As $a_{n} \geq 0$, growing the amount of antennas at each BS will definitely improve the coverage probability. It can be found from (19) that $a_{n}>a_{n+1}$ for $n \geq 0$, this implies that the coverage probability decreases by including one more antenna at the BS. $\eta_{E E}(M)$ denotes the $\mathrm{EE}$ as a function of $M$ antennas at each BS and this can be expressed by substituting (18) in (6):

$$
\begin{array}{r}
\eta_{E E}(M)=\frac{\lambda_{M B S} \lambda_{u s e r} \sum_{n=0}^{M-1} a_{n} \log _{2}(1+\bar{\gamma})}{\lambda_{\text {user }}\left[\frac{1}{\eta_{A E 1}} P_{t p 1}+M P_{c p 1}\right]} \\
+\lambda_{M B S}\left[\frac{1}{\eta_{A E 2}} P_{t p 2}+M P_{c p 2}\right] \\
+\left(\lambda_{F B S}+\lambda_{M B S}\right) P_{n t p}
\end{array}
$$

A non-zero optimal number of antennas at each BS can be denoted by $M^{*}$ and optimizes the EE. Higher values of $M$ reduces the EE for $M>M^{*}$, whereas for $M<M^{*}$, exploiting further antennas improves EE. The impact of $M$ antennas on the EE can be observed from the following property:

Lemma 3: The numerical value of $M^{*}$ is the highest whole number which can become lesser compare to the solution of the following equation:

$$
S(M)=\frac{p\left(\frac{1}{\eta_{A E 1}} P_{t p 1}\right)+\frac{1}{\eta_{A E 2}} P_{t p 2}+P_{n t p}}{p P_{c p 1}+P_{c p 2}},
$$

where $S(M) \triangleq \frac{p_{c o v}(M)}{a_{M-1}}-M$.

Proof : From (14), it can be shown that this is impossible that the two inequalities, $\eta_{E E}(M) \leq \eta_{E E}(M-1)$ and $\eta_{E E}(M) \leq \eta_{E E}(M+1)$ to be held concurrently; the latter implies that both decreasing and then increasing response of the EE is never possible to be achieved as we keep enhancing the number of antennas at the BS. Besides, we have $\lim _{M \rightarrow \infty} \eta_{E E}(M)=0$ and $\eta_{E E}(1)>0$. Based 
on the entire facts, the following two cases can be considered: 1) the $\mathrm{EE}$ deteriorates with $M$, thus exploiting the BSs of a single-antenna types is even more energy efficient than the BSs of multiple-antenna types; 2) exploiting the BSs of multiple-antenna types can produce even greatest EE than BSs of a single-antenna types, but only at the optimal value of $M$. To address the optimal condition, i.e., $M^{*}$, we choose the following inequalities as:

$$
\left\{\begin{array}{l}
\eta_{E E}\left(M^{*}\right) \geq \eta_{E E}\left(M^{*}-1\right), \\
\eta_{E E}\left(M^{*}\right) \geq \eta_{E E}\left(M^{*}+1\right) .
\end{array}\right.
$$

Substituting (14) into (16), we get:

$$
\begin{aligned}
\frac{\sum_{n=0}^{M^{*}-1} a_{n}}{a_{M^{*}-1}}-M^{*} & \leq \frac{p\left(\frac{1}{\eta_{A E 1}} P_{t p 1}\right)+\frac{1}{\eta_{A E 2}} P_{t p 2}+P_{n t p}}{p P_{c p 1}+P_{c p 2}} \\
& \leq \frac{\sum_{n=0}^{M^{*}} a_{n}}{a_{M^{*}}}-\left(M^{*}+1\right) .
\end{aligned}
$$

For $S(M) \triangleq \frac{p_{c o v}(M)}{a_{M-1}}-M$ the numerical value of $M^{*}$ is the highest integer which becomes less than the solution of (15).

As $S(M)=\frac{1}{a_{M-1}} \sum_{N=0}^{M-2}\left(a_{n}-a_{M-1}\right)$, it implies that $S(M)$ is a growing function with $M$. Now, if we could exploit FBSs and MBSs with a lower $P_{c p 1}$ and $P_{c p 2}$ respectively then the optimal number of antennas at the BSs would have higher and improved performance out-turns of the EE could be seen. Applying BSs of a single- antenna types can produce more $\mathrm{EE}$ in contrast to applying BSs of multiple- antenna types. In this regard, we can see the boundary condition from (22) as $P_{t p 1}$ or $P_{t p 2} \geq \frac{x_{0}\left(p\left(\frac{1}{\eta_{A E 1}} P_{t p 1}\right)+\frac{1}{\eta_{A E 2}} P_{t p 2}+P_{n t p}\right)}{1+\left(x_{0}+x_{1}\right) p}$, where the right hand portion of the condition is a monotonic function of $p$, it implies that if the boundary constraint

$$
P_{c p 1} \text { or } P_{c p 2} \geq \max \left(\begin{array}{c}
x_{1}\left(\left(\frac{1}{\eta_{A E 1}} P_{t p 1}\right)\right. \\
+\frac{1}{\eta_{A E 2}} P_{t p 2} \\
x_{1} P_{n t p}, \frac{\left.+P_{n t p}\right)}{1+x_{0}-x_{1}} \\
\\
\triangleq \bar{\gamma} P_{n t p}
\end{array}\right)
$$

is satisfied for both types of BSs and any $\lambda_{\text {user }}$, then the EE level of a single-antenna type BSs would have been higher in contrast to the multiple-antenna type BSs. Hence, BSs of multiple-antenna types are superior to achieve energy efficient networks only when $P_{c p 1}$ or $P_{c p 2}<\bar{\gamma} P_{n t p}$.

\section{NumERICAL RESUlTS AND DisCUSSION}

In this section, we demonstrate that interpretative simulation results are in line with the theoretical results in the PPP-based model. Fig. 1a plots the coverage probability as a function of FBS density denoted by $\lambda_{F B S}$ at different values of $M$ keeping the user density $\lambda_{\text {user }}=10^{-2} \mathrm{~m}^{-2}$ fixed. We find that the coverage probability increases with the increase of $\lambda_{F B S}$ or $M$. Precisely, a significant gain improvement can be noticed for the shift of $M$ from 4 to 7 , whereas achieved gain becomes lower for the shift of $M$ from 4 to 1 . From Fig. 1a, we also find that both the analytical results and simulation results have the same trend. For the fixed adaptation of transmit power, coverage probability decreases for a reference value of $M$ in the lower FBS density region compared to that of the presented work in [8] and upper FBS density region illustrates smooth and in-line response with the results that have shown in [8].

Fig. 1b depicts the EE as a function of FBS density denoted by $\lambda_{F B S}$ at different values of $\mathrm{M}$ keeping the femto user density $\lambda_{\text {user }}=10^{-2} \mathrm{~m}^{-2}$ fixed. The network settings are different in comparison with Fig. 1a. Here, $\eta_{A E 1}=0.35$, $P_{t p 1}=7.3 \mathrm{~W}$ and $P_{c p 1}=38 \mathrm{~W}$ are taken into account for a FBS in the base station power model. By replacing all these typical values, it can be seen that the boundary constraint (23) is satisfied, which implies that deployment of a single-antenna type FBSs is superior in terms of EE than the multi-arrangement-type FBSs. In particular, a 
significant deterioration in the EE can be noticed for the shifting of $M$ from 1 to 4 and it is deteriorated even more for the shifting of $M$ from 4 to 7 , although the response of both the analytical results and simulation results are all that remain same. From Fig. 1b, it can be seen that optimal FBS density for $M=1$ is around $0.7^{-2} \mathrm{~m}^{-2}$ for both analytical and simulation results. Fig. 1(b) also shows that the response of the network for $\mathrm{EE}$ in accordance with the consideration of fixed $\eta_{A E}$, where EE decreases rapidly in the upper FBS density region compared to the work presented in [8] is only because of the extra loss produced due to the extention of the layer from one to two.

\section{CONCLUSION}

We addressed a novel set of numerical outcomes to analyze performance of a PPP based random femto-cellular networks. Based on all these outcomes, the impacts of $\lambda_{F B S}$ and $\lambda_{\text {user }}$ at the BS have been probed on the EE. The outcomes observed in this shorter version of the paper are particularly useful for today's multi-arrangement antenna transmission based dual-layer cellular networks.

\section{REFERENCES}

[1] Joydev Ghosh (2019): Energy Efficiency Analysis by GameTheoretic Approach in the Next Generation Network, IETE Technical Review, DOI: 10.1080/02564602.2019.1620139

[2] I. Budhiraja, S. Tyagi, S. Tanwar, N. Kumar and M. Guizani, "cross layer NOMA interference mitigation for femtocell users in 5G environment," IEEE Transactions on Vehicular Technology, Vol. 68, No. 5, May 2019.

[3] Joydev Ghosh, Dushantha Nalin K Jayakody, Marwa Qaraqe, "Downlink capacity of OFDMA-CR based 5G femtocell networks," Physical Communication, vol. 29, pp. 329-335, August 2018.

[4] I. Budhiraja, S. Tyagi, S. Tanwar, N. Kumar and N. Guizani,"Subchannel Assignment for SWIPT-NOMA based HetNet with Imperfect Channel State Information," 15th International Wireless Communications Mobile Computing Conference (IWCMC), 2019

[5] I. Budhiraja, S. Tyagi, S. Tanwar, N. Kumar and M. Guizani,"CR-NOMA Based Interference Mitigation Scheme for 5G Femtocells Users," IEEE Global Communications Conference (GLOBECOM) 2018.

[6] Joydev Ghosh "Interrelationship between energy efficiency and spectral efficiency in cognitive femtocell networks: A survey," Pervasive and Mobile Computing, Volume 59, October 2019.

[7] J. Ghosh,"A trade-off between Energy Efficiency and Spectral Efficiency in macro-femtocell networks," IEEE Transactions on Vehicular Technology, DOI: 10.1109/TVT.2019.2937920.

[8] Chang Li, Jun Zhang, and Khaled B. Letaief,"Throughput and Energy Efficiency Analysis of Small Cell Networks with Multi-Antenna Base Stations," IEEE Transactions on Wireless Communications, VOL. 13, NO. 5, MAY 2014.

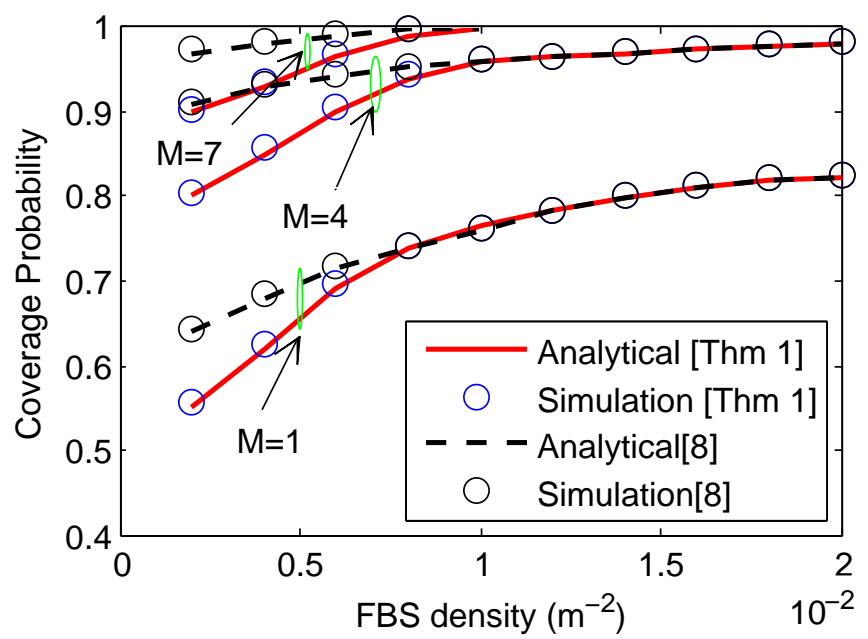

(a)

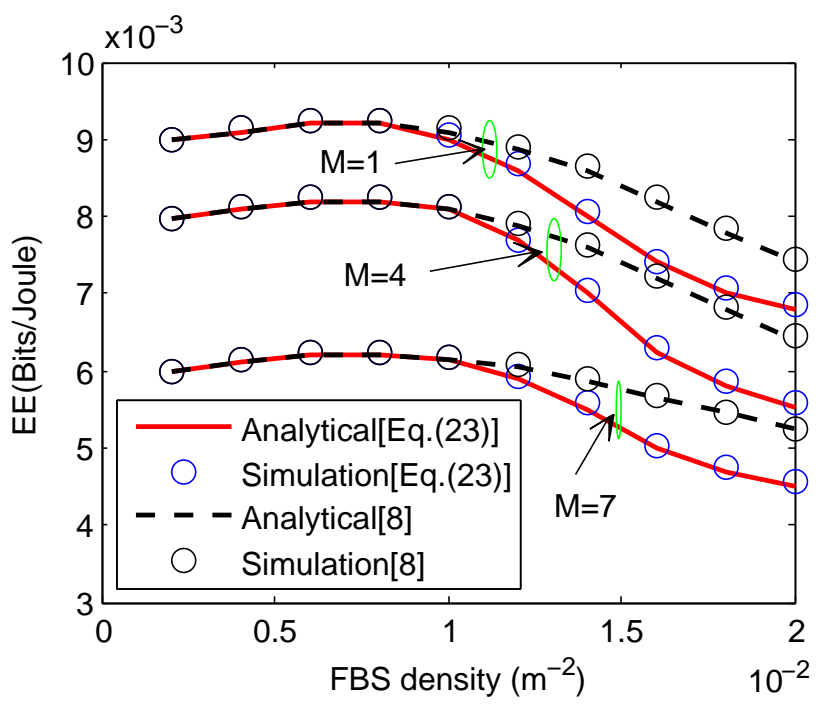

(b)

Figure 1: Coverage probability vs. $\lambda_{F B S}$ for different number of antennas at the FBS, where $\alpha=3$, $\bar{\gamma}=1, \lambda_{F U}=10^{-2} \mathrm{~m}^{-2}, P_{t p}=7.3 \mathrm{~W}$, Bandwidth $=400 \mathrm{KHz}, N_{0}=-96.5 \mathrm{dBm}$ (Fig. 1a), EE vs. $\lambda_{F B S}$ for different number of antennas at the FBS, where $\alpha=3, \bar{\gamma}=1, \lambda_{F U}=10^{-2} m^{-2}, \eta_{A E}=0.35$, $P_{t p}=7.3 \mathrm{~W}, P_{c p}=38 \mathrm{~W}$, Bandwidth $=400 \mathrm{KHz}$, $N_{0}=-96.5 \mathrm{dBm}$ (Fig. 1b).

[9] Trupil Limbasiya, Debasis Das, "Secure message confirmation scheme based on batch verification in vehicular cloud computing,"Physical Communication, Volume 34, pp. 310-320 June 2019.

[10] J. Xu and L. Qiu, "Energy efficiency optimization for MIMO broadcast channels," IEEE Trans. Wireless Commun., vol. 12, no. 2, pp. 690-701, Feb. 2013.

[11] D. Commenges and M. Monsion, "Fast inversion of triangular Toeplitz matrices," IEEE Trans. Autom. Control, vol. 29, no. 3, pp. 250-251, Mar. 1984.

[12] S. Lee and K. Huang, "Coverage and economy of cellular 
networks with many base stations," IEEE Communications Letters, vol. 16, no. 7, pp. 1038-1040, July 2012.

[13] C. Isheden, Z. Chong, E. Jorswieck, and G. Fettweis, "Framework for link-level energy efficiency optimization with informed transmitter," IEEE Trans. Wireless Commun., vol. 11, no. 8, pp. 2946-2957, Aug. 2012.

[14] J. G. Andrews, F. Baccelli, and R. K. Ganti, "A tractable approach to coverage and rate in cellular networks," IEEE Trans. Commun., vol. 59, no. 11, pp. 31223134, Nov. 2011.

[15] M. Shafi at al "5G: A Tutorial Overview of Standards, Trials, Challenges, Deployment, and Practice," IEEE Journal on Selected Areas in Communications, vol. 35, no. 6, pp. 1201 1221, June 2017.

[16] A. Vecchio, "A bound for the inverse of a lower triangular Toeplitz matrix," SIAM J. Matrix Anal. Applicat, vol. 24, no. 4, pp. 1167-1174, 2003. 